\title{
A Solution for Robotized Sampling in Wastewater Plants
}

\author{
Edmundo Guerra, Yolanda Bolea, Antoni Grau \\ Automatic Control Department \\ Technical University of Catalonia, UPC \\ Barcelona, Spain \\ \{edmundo.guerra;yolanda.bolea;antoni.grau\}@upc.edu
}

\author{
Rodrigo Munguía \\ Department of Computer Science \\ University of Guadalajara,CUCEI \\ Guadalajara, Mexico \\ Rodrigo.munguia@upc.edu
}

\author{
Javier Gamiz \\ AQUOLOGY \\ Barcelona, Spain \\ jgamiz@aqualogy.net
}

\begin{abstract}
This work presents a solution to automatize the water sampling process of outdoor basins in a wastewater treatment plant. The system proposed is based on the utilization of collaborative robotics: a team of an UAV and a terrestrial robotic platform make a route along the plant collecting and storing the water samples. The architecture of the designed system is described in terms of functional blocks, and implementation details including software frameworks and hardware on the UAV are provided. As the objective of the system is industry levels of robustness and performance, the UAV use is minimized and subjected to control from the robotic ground platform, reducing risks associated with autonomous UAV. To conclude, results from experiments performed to validate the viability of the system and study several design decisions are presented and briefly discussed, including: estimation of the accuracy of several GNSS technologies on the plant, viability of the landing operation over a mobile robotic platform and controlling a quadrotor over waters.
\end{abstract}

Keywords-Wastewater plant, UAV, sampling.

\section{INTRODUCTION}

Industrial development has been largely driven by technical innovations, with better, cheaper and more efficient ways to produce goods and services made possible by them. The automatization of procedures has made the human physical effort largely redundant on many industrial processes, freeing man from heavier tasks. Still, many tasks require human presence, given the versatility and prowess of human cognition capabilities. These tasks generally remain associated with supervisory and management roles, where specialist knowledge and skills which takes years to learn are required. This kind of expertise is hard to model mathematically, thus, the human presence cannot be really substituted, but it can be assisted in several ways [1].

Robots have been present in industry as part of automated system for a long time. Moreover, robotic vehicles have become a critical component in many automated storage and logistics services, with carts and forklifts transporting freight only with human supervision. The last addition to the field of autonomous robotic vehicles is a wide variety of unmanned aerial vehicles (UAV), which already have a strong presence in the research community, the audiovisual sector and the spatial and surveying industry [2]. Several world class enterprises are putting great efforts to introduce them as part of logistic and distribution systems. Still, many sectors in the industry remain indifferent to the capabilities of autonomous UAV given the risks associated to having robotic devices flying by themselves. This means that only under human supervision the UAV's are being slowly introduced in the wider industry, especially in surveying and monitoring tasks. In these applications, their mobility and freedom of movement means reductions in costs and risks for staff.

In many industries surveying and monitoring tasks imply sampling of products at different steps. This is the case of wastewater treatment plants. An average water treatment plant has to control and monitor several processes, including the waters produced at each treatment step; which translates into analyzing several basins and tanks frequently. These analyses require availability of recent samples obtained from the tanks; and procuring them is a cumbersome, systematic, frequent task, the kind of task that could benefit from an automated process. The structure of an outdoor water treatment facility generally includes open air basins. In this kind of open environment, UAV can travel freely through most of the installation, with little worries about complex navigation and obstacle avoidance.

In this work we present an automated system designed with the goal of automate water sampling tasks in a treatment plant. The system will use to autonomous vehicles, a robotic ground platform and a UAV, which will collaborate to collect batches of water samples in several tanks. Section 2 discusses risks and requirements of the water sampling problem, and describes the plant targeted by the system. The following section describes the general architecture of the system, detailing how the system is controlled and supervised; the hardware and software structure of the robotic UAV built; and the functional design criteria and some of the hardware considered for designing the ground robotic platform. Fourth section describes and comments several preliminary experiments performed to test the viability of the system and several design decisions. Finally, the conclusions discuss the next step in the testing and development of the system.

\section{WASTEWATER SAMPLING ON TREATMENT PlANTS}

\section{A. Evaluation and risks of water sampling}

Evaluation of processed waters in a wastewater treatment facility requires testing for measurement of several factors, including chemical properties of the water and both biological 
and inorganic pollutants. The main values that must be analyzed include $\mathrm{pH}$, biological oxygen demand (BOD, measured through the 5 days test, $\mathrm{BOD}_{5}$ ), chemical oxygen demand (COD, nitric nitrogen, ammonia, suspended solids, phosphorous, metallic pollutants, and many more. Generally this means taking multiple samples, with different requisites: nitric nitrogen must be tested within 2 hours to avoid reactions, $\mathrm{DOB}_{5}$ requires the sample temperature to be controlled, microbiological testing forbids the samples from sunlight exposition, etc... In plants were the testing are required within a given periodicity (some values are to be tested daily according to normative, this means meticulous planning and availability of human and technical resources just for the sample taking process.

In most of the values and procedures to analyze both waters and wastewaters, one of the key features is the homogeneity of the sampling. This is desired in order to guarantee the representability of the sampling, so that it can be concluded that the results of the analysis will be accurate and representative of the waters present in the tank. The tanks present in wastewater treatment plants present slow dynamics and flow speeds compared to the dilution processes, which helps homogenize concentration of pollutants. At the same time they are generally of big proportions, meaning that the diffusion and dilutions processes are distributed along a great body of water, and they act as a buffer with delays: the tank may contain waters which have been subjected to the different treatment and dilutive processes during uneven periods of time. Thus, in order to sample a wastewater tank to obtain significant analytics, multiple samples must be collected a different points. This can become a difficult process in bigger tanks, which could require special equipment or installations to reach inner points on wider deposits.

Wastewater treatment facilities present additional challenges with respect to the sampling tasks. Not only their structure means that reaching certain points require scaffolding or similar aiding structures (which in many countries would require presence of additional staff for the sake of security), but can present several risks, such as the presence of noxious gasses or substances. Other factors to be accounted include the procedural requirements of sampling to produce valid results, such as requirements of preservation for the samples (time until analysis is performed, temperature and exposition to light admitted, etc...), which can put pressure on staff, resulting in increased risk-taking decisions. All the problems described can be further exacerbated by adverse weather on outdoor installations and or working during night or other periods that reduce concentration and productivity.

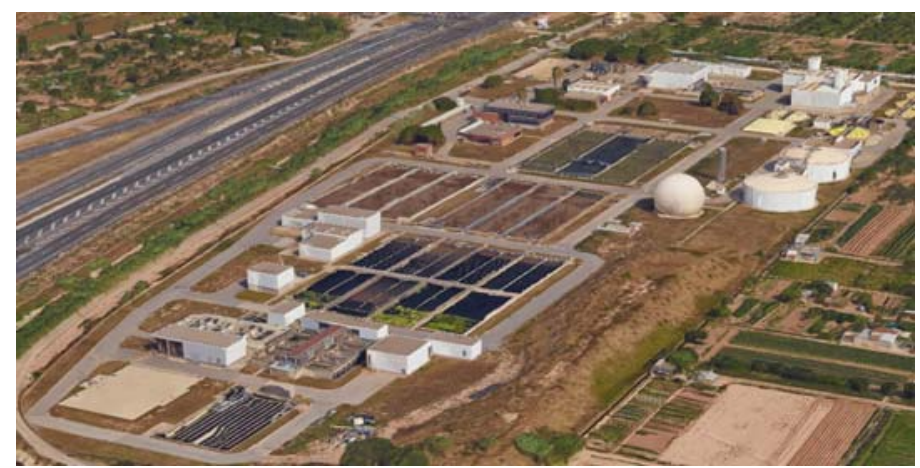

Figure 1: Wastewater treatment plant at San Feliu de Llobregat, Barcelona.
All these risks must be managed, and there are several means: improving methodologies, installations, equipment, staff formation, extra labor force, etc. Most of these ways to manage risks and challenges associated to sampling procedure share two distinct features: they require additional inversion and they reduce but fail to remove the risks and failures due human practice.

\section{B. Plant description}

For the proposed system, we considered a medium wastewater processing plant which treats up to $72000 \mathrm{~m}^{3}$ of water, or the equivalent of 320000 inhabitants and their economic activities. This wastewater plant (Fig. 1) serves the sanitation system in Sant Feliu of Llobregat, $10 \mathrm{~km}$ from Barcelona, in the Basin of Llobregat River. As an area known for its industry, the plant deals mainly with waters from two origins: domestic sewage, used in residential housing, offices, shops and any other space without special pollutants; and pretreated industrial water, thrown after a treatment in the facility to remove specifics pollutants.

The finality of this plant is to return the wastewater and pluvial water to the environment in the best conditions as possible according the quality standards specified in the present regulation. To achieve this objective, in the plant the water is passed through different processes consisting on filtering it, decanting it or accelerating the degradation of dissolved organic matter. With those actions, the pollutants are removed from the water such as floating solids (plastic, non-dissolved papers...), sands, fats and organic matter. This means that the plant presents 17 outdoor open air basins, covering a surface over $11200 \mathrm{~m}^{2}$.

\section{SYSTEM ARCHITECTURE}

The system proposed to automatize the water sample collection process aims to maximize the sample collecting capabilities in terms of accessibility to the desired point of interest and minimizing the efforts and risks taken by the human staff. This is achieved by giving the chance to collect batches of samples without even leaving the laboratory.

Two different autonomous agents will cooperate in the sample collection process: a quadcopter UAV, acting as sample collector, and a robotic platform acting as sample carrier. This means that the UAV will generally remain on the sample carrier platform, and take flight to capture a sample on the points elected by the staff once the carrier has completed the approximation to the relevant basin. The sample collector will take a water sample on the coordinates indicated by the carrier, and go back to it, where the sample is to be stored, and sample container in the UAV will be replaced.

\section{A. System architecture and communications}

The general architecture and proceeding of the system can be seen in fig.2, showing how it has been segmented to better fit deployment into an industrial plant: the laboratory staff uses the scheduling the Scheduling and Control module interface to generate a collection order. This collection order details how many samples on each basin must be taken, on which area of 


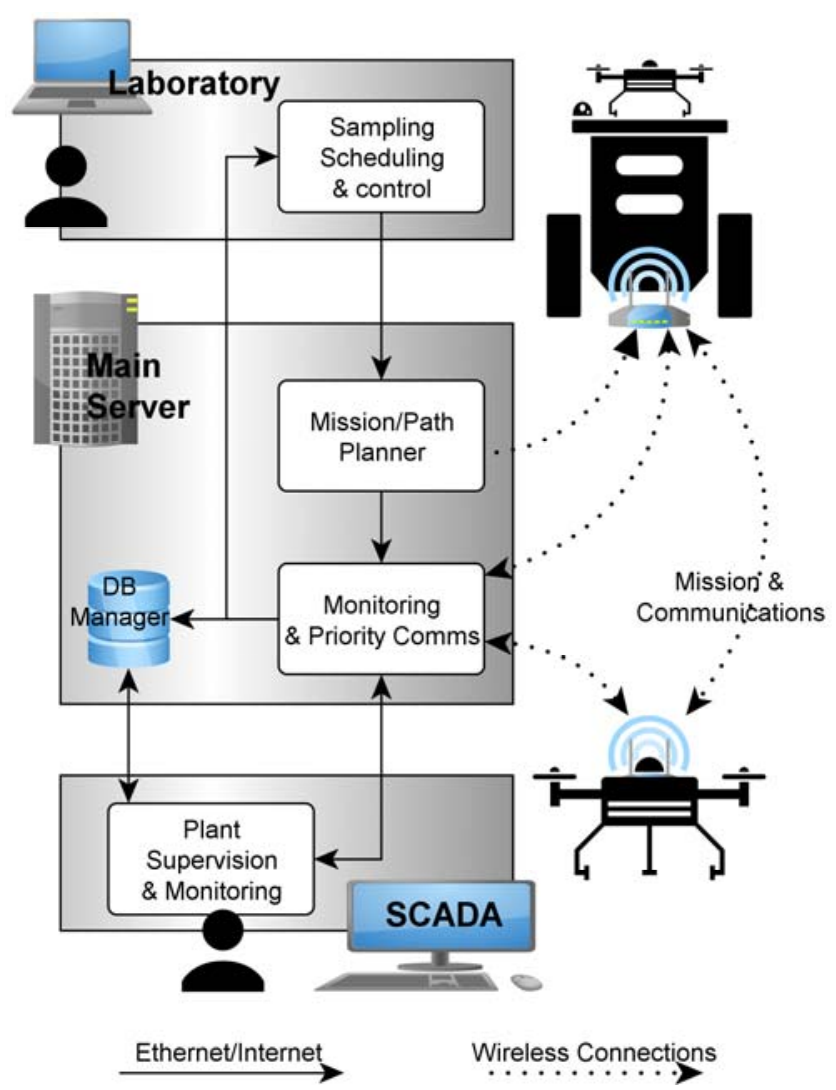

Figure 2: Proposed system distribution and main communications diagram

the basin is each sample to be collected, and when is the sampling process to start.

Once the order is generated (a list of GPS coordinates with additional options and timing data), it is processed on the main server of the system, where a mission path will be produced. This mission path specifies a route that will be taken by the sample carrier platform, including several stop points. In these stopping points, the carrier will transmit to the sample collector UAV the data for a collection mission. The collection mission normally consists in some brief height indications along the coordinates of the point where the sample must be taken.

The mission path planner use a 2-dimensional grid model of the outdoors of the plant to compute a path for the carrier drone, including stopping points and the samples to be taken. Using a grid model of the plant allows reducing greatly the computational costs of the process, and enriching the map modelled with additional data, such as scheduling the accessibility of a given space or area. The map is processed through an energy minimization planning approach, loosely based on [3], accounting several ordered criteria:

1. all the samples of the same basin/tank must be captured consecutively
2. minimize the expected flight effort for the collector UAV (heuristically computed as if the trajectory was made of orthonormal movements)

3. minimize penalizations from restricted accessibility areas

4. minimize the distance travelled by the sample carrier

All the criteria (mainly thought for 2 and 3) are subjected to parametrizable tolerance and checks in order to avoid nonsensical cases, e.g.: the sample carrier moving less than $1 \mathrm{~m}$ between to stops for the UAV to take flight.

All the data generated by the system is logged and stored, including the collection orders and mission paths, as any alert and system status surveying data. The communications with the autonomous vehicles are carried through $4 \mathrm{G}$ in order to enable visual supervision through streaming (for the UAV), while the rest are to be deployed through Ethernet (although the segmented architecture enables working remotely: Internet, VPN's, etc...). The communications between the carrier and the sample collector are performed through ZigBee, though they also deploy WiFi transmitter for development/debugging purposes. The priority communications, bundled with the supervision and surveying, comprise all the signaling and procedures that being unplanned are capable of overriding the normal operations, include the emergency recall protocol. For the UAV, the recall protocol includes a set of fallback points coordinates: first it will try to go back and land in the sample carrier, even if it is inoperative, as the recall notification may include its last known coordinates. If that is not possible, the UAV may try to reach another given fallback point. If the drone battery drops below certain thresholds, it will just search for a clear patch of ground (avoiding waters/pipes/other obstacle structures through visual inspection) and try to land. This is a last resort as it may damage the sample capturing device.

\section{B. Sample collector drone architecture}

The sample collector UAV is a custom quadcopter based partially on the Tarot X4 frame, measuring $96 \mathrm{~cm}$ radius, four 18', propellers with T-Motor MN4014 actuators. A PIXHAWK kit modified to support a pair of 6S 10000mah batteries is used as FMU (Flight Management Unit), including

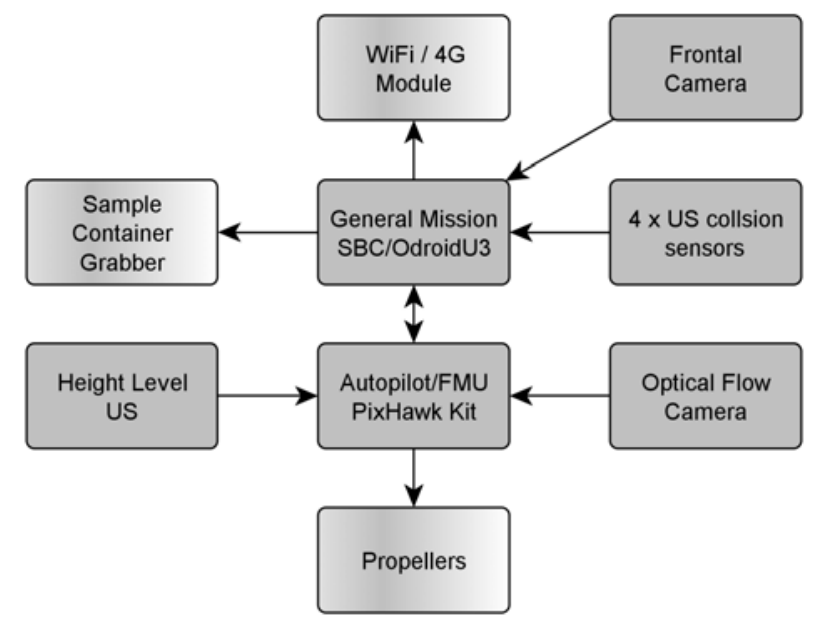

Figure 3: Collector UAV hardware communications diagram 


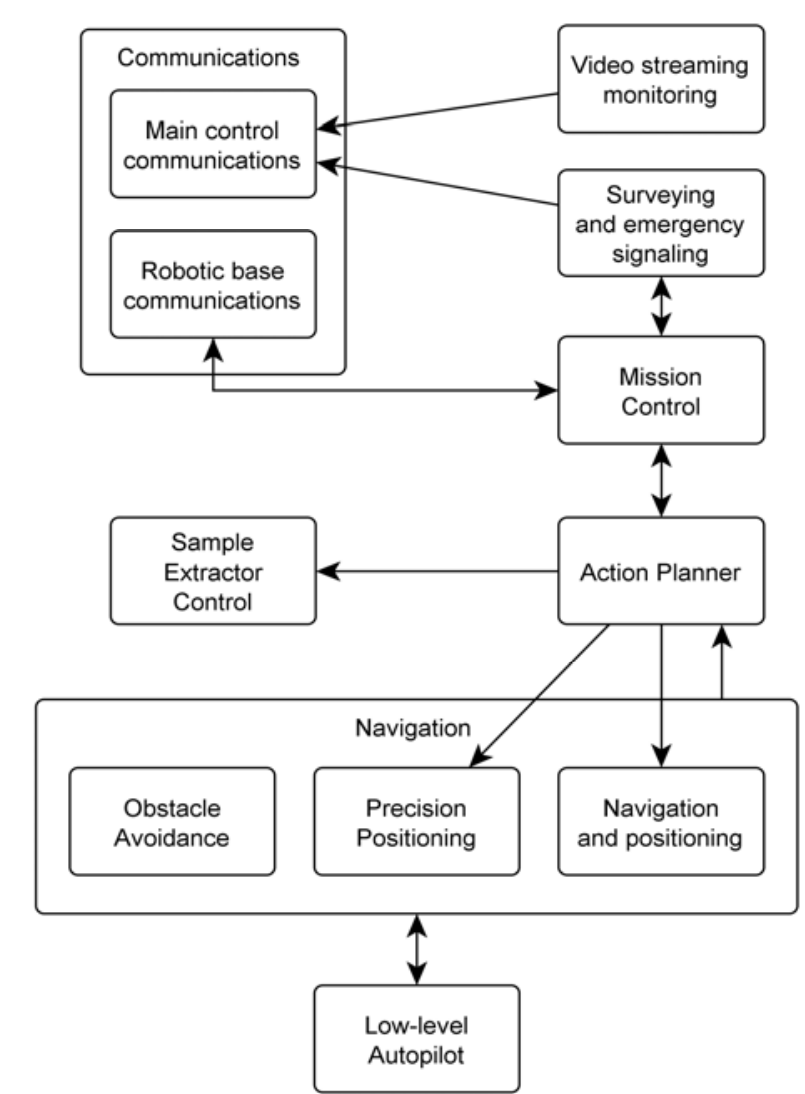

Figure 4: Sample collector UAV block diagram

an inertial unit, a SBAS (EGNOS network), the ESC, etc An Odroid U4 SBC is used to deal with all non-critical for navigation inputs and procedures. This low-power SBC deploys a Exynos5522 octa-core dice, with both ARM Cortex A15 and A7, and a OpenCL compliant GPU. In terms of sensors, besides the FMU inertial unit, an optical flow camera (PX4FLOW) with a beam sonar is deployed facing downward, and a front facing USB 3.0 5MP camera (although normally set at 640x480 pixels at $30 \mathrm{fps}$ ). To avoid collision, a set of 4 MaxBotix ultrasonic sensors are deployed at a horizontal plane. The FMU runs the PX4 flight stack [4], while the SBC hardware runs an Ubuntu 14 for ARM distribution, with ROS [5] Jade, and support of MavLink for communications. Fig. 3 details the communications of the components at hardware level.

The UAV hardware weights about $4270 \mathrm{~g}$, with a maximum theoretical lift of $13900 \mathrm{~g}$. This puts the UAV around a 0.3 weight/lift ratio. The sample collector UAV's design is aimed at producing a container and grabber with weight below $300 \mathrm{~g}$. This would allow collecting even $1000 \mathrm{ml}$ samples keeping the weight/lift ratio around 0.4 .

In terms of operation, the collector UAV follows the block diagram in fig. 4. The communications are divided into two blocks, depending on the channel and target: Mavlink over 4G is used for streaming video (if activated), and communicating with the monitoring and priority communications modules, in order to guarantee the capabilities to supervise the operations and keep safety measures active. The mission control module, running on the SBC, receives the collection mission from the sample carrier platform, which is processed by the action planner, which translates in into a simple trajectory for the FMU. As the sample container sinks into a basin, it fills itself, increasing the weight of the UAV by less than $10 \%$ (under the assumption of taking $500 \mathrm{ml}$ water samples).

During flight, the UAV is positioned through EGNOSenhanced GPS (see Section IV.A). To approach the water surface and land over the sample carrier other sensors are required, thus the precise positioning algorithms are used. In the landing case, the robotic sample carrier presents several fiducial markers to easily compute the relative pose of the UAV in real-time, which enables the insertion of the sample container into a socket, similarly to an assisted peg in the hole operation. The action planner will then release the container through its control module, so that the carrier can replace it with a clear one if required, or simply store it to keep the sample isolated from the environment.

Currently the autonomous navigation of the drone is largely based on the EGNOS localization and sensory input to avoid obstacles. This requires the assumption of an open environment, so that with minimal data and knowledge (passed through by the sample carrier 'sample mission') the UAV can navigate and find the way easily. Still this option is better suited that the alternatives, be it deploying a full sensory autonomous navigation suite (which sensors like stereo-vision, RGB-D, LiDAR/RADAR), and/or full accurate modelling of the environment, with enough computational power to process any of the alternatives.

\section{Sample carrier and collection process design.}

The sample carrier is based on a Seekur Jr robotic platform from Adept Mobile Robotics. The base platform has been fitted with frontal and backward Hokuyo LRF, frontal stereo camera and a SBC able GPS. These specifications can be subjected to modifications as the design go on.

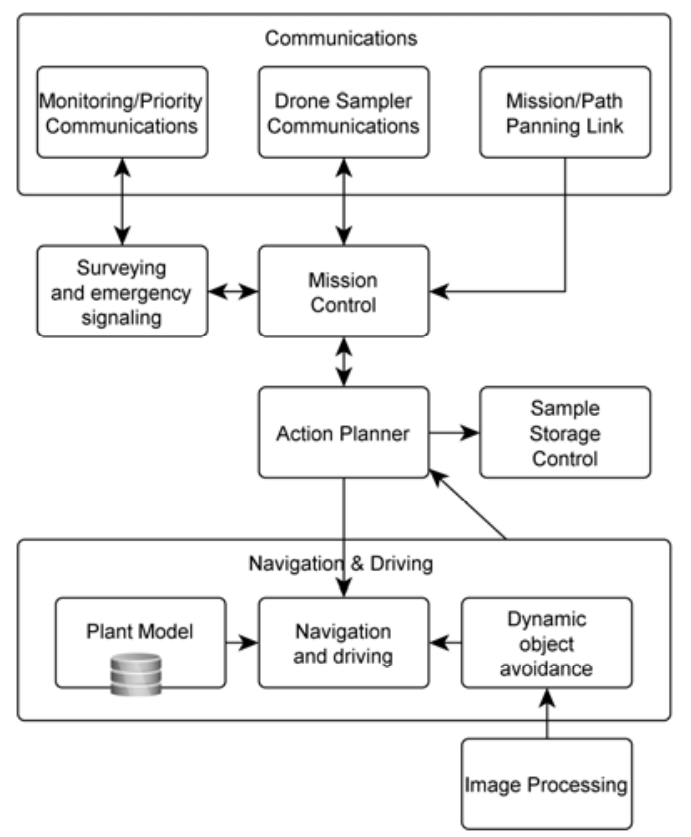

Figure 5:Sample carrier robotic platform block diagram 
The sample collection container and the receiver mechanism are currently under mechanical design, while the functional architecture is show at diagram fig. 5. Though there have been successful implementations of UAV with manipulator arms able to retrieve and operate small loads [6], this would mean a cost and payload overhead, as our sample collector does not need flexible precise manipulation. As such, the approach taken by the design team (with participation of industry partners) is a container with two high rods which catch into the drone. After the collector UAV has landed into the carrier, these rods can be released with the action of a small stepper motor, thus releasing the sample container into a rotary drum. This rotary drum is in a height-controlled platform that goes down between 7 and $10 \mathrm{~cm}$, so that the rotary drum can spin placing a clean container in position. After the UAV has finished readying the rod receiving clasps, the platform inside the carrier raises again, attaching the container to the collector UAV.

\section{PRELIMINARY FLIGHT RESULTS AND DESIGNS.}

Multiple tests have been performed to validate the viability of the project, and help with several design decisions.

\section{A. Localization and positioning}

One of the most challenging aspects on the proposed system is the positioning problem. While there are plenty of available solutions, the size of the installation and the outdoor environment makes any solution based on adding support elements expensive and potentially hard to maintain.

TABLE I. LOCALIZATION TECHNOLOGIES TESTED

\begin{tabular}{|c|c|c|c|}
\hline \multirow{2}{*}{$\begin{array}{c}\text { Sensor } \\
\text { Technology }\end{array}$} & \multirow{2}{*}{$\begin{array}{c}\text { Nominal } \\
\text { errors }\end{array}$} & \multicolumn{2}{|c|}{ Experimental error ${ }^{a}$} \\
\hline & & Avg. & $\sigma^{2}$ \\
\hline GPS & $15 \mathrm{~m}$ & $8.9 \mathrm{~m}$ & $3.2 \mathrm{~m}$ \\
\hline $\begin{array}{l}\text { GPS with } \\
\text { EGNOS }\end{array}$ & $3 \mathrm{~m}$ & $1.3 \mathrm{~m}$ & $0.4 \mathrm{~m}$ \\
\hline RMCB system & $*^{\mathrm{b}}$ & $3.4 \mathrm{~m}$ & $2.1 \mathrm{~m}$ \\
\hline
\end{tabular}

Table 1 shows the results obtained in preliminary in-site experiments to evaluate the localization systems. Several reference points originally obtained using differential GPS were used to estimate a ground truth for the experiment. The RMCB deployed 6 beacons of the TI eZ430-RF2500, based on the CC2530 chip, covering a $40 \mathrm{~m}$ per $20 \mathrm{~m}$ area. The errors obtained with EGNOS enhanced GPS were well below expectations, though their repeatability under other circumstances cannot be assured, even in the same site. Still, they are accurate enough so that even assuming worse errors under other circumstances, the system will perform adequately, as the critical operations are mainly guide with vision sensors.

The wireless network localization literature found described errors proportionally lower that experimented [7], [8]. Besides, these kinds of approaches generally assume a planar environment, which would not be the case if used in the proposed system.

\section{B. Sample collector UAV flight}

The take-off and landing over on a surface with fiduciary markers problems have been extensively dealt in the literature. Experimental tests of a landing operation while inserting a fixed cylinder in a hole with tolerance were performed using a smaller prototype drone. This custom-built drone (see Fig.6) landed with a $10 \mathrm{~cm}$ diameter cylinder fixed beneath, inserting it into a $12 \mathrm{~cm}$ overture on a surface.

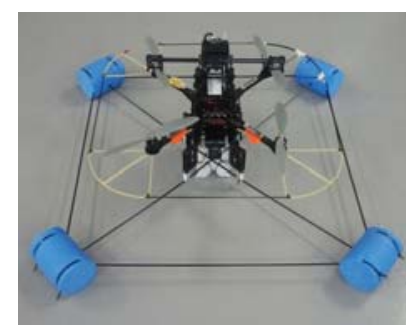

Figure 2: Drone use in landing while inserting tests.

Another set of experiments was simulated to evaluate the viability of the sample container sinking operation. The simulations, implemented on Simulink [9], [10], were aimed at testing how the controllers would interact with an opposing upwards force when trying to sink the sample container, and the effects of the increasing variable payload on the stability of the system. The opposing force experiment was modelled as if the UAV tried to 'land' on a compression spring, whose force decreases if certain compression threshold is surpassed (to emulate the behavior of the floatability opposing force, which decreases once the container starts to fill). The increase in the mass was assumed to be uniform, and for the sake of simplicity ignored the fluid dynamics.

\section{CONCLUSIONS}

An industrial system to automatize the water sampling processes on outdoor basins in a wastewater treatment plant has been presented. The architecture of the whole system has been described, while specifications at hardware level have been presented for those elements designed completely. To validate the viability of the approach several experiments have been performed, focusing on the most critical and potentially problematic aspects. To evaluate the localization issue, several positioning technologies have been tested on site, with SBAS enhanced GPS (with EGNOS) offering the best results. From the UAV control point of view, there are two critical mission steps: landing into the sample carrier inserting the container, and drowning the container into the water and keeping the UAV stable. Both real and simulated experiments have been carried to prove the feasibility of these processes. Still, further experimentation with actual water bodies is required to tune controllers and evaluate limitations. Once the sample carrier is fully designed, experiments on autonomous navigation on site are to be performed, including carrier navigation and localization and collector UAV landing. 


\section{ACKNOWLEDGMENT}

This research was funded by Spanish Science ministry project CICYT DPI2013-42458-P (Robot-Int-Coop).

\section{REFERENCES}

[1] C. Heyer, 'Human-robot interaction and future industrial robotics applications', in 2010 IEEE/RSJ International Conference on Intelligent Robots and Systems (IROS), 2010, pp. 4749-4754.

[2] J. T. K. Ping, A. E. Ling, T. J. Quan, and C. Y. Dat, 'Generic unmanned aerial vehicle (UAV) for civilian application-A feasibility assessment and market survey on civilian application for aerial imaging', in 2012 IEEE Conference on Sustainable Utilization and Development in Engineering and Technology (STUDENT), 2012, pp. 289-294.

[3] N. Ganganath, C. T. Cheng, and C. K. Tse, 'A Constraint-Aware Heuristic Path Planner for Finding Energy-Efficient Paths on Uneven Terrains', IEEE Trans. Ind. Inform., vol. 11, no. 3, pp. 601-611, Jun. 2015.

[4] L. Meier, D. Honegger, and M. Pollefeys, 'PX4: A node-based multithreaded open source robotics framework for deeply embedded platforms', in 2015 IEEE International Conference on Robotics and Automation (ICRA), 2015, pp. 6235-6240.
[5] D. Schleicher, L. M. Bergasa, R. Barea, E. Lopez, M. Ocana, and J. Nuevo, 'Real-time wide-angle stereo visual SLAM on large environments using SIFT features correction', in IEEE/RSJ International Conference on Intelligent Robots and Systems, 2007. IROS 2007, 2007, pp. 3878-3883.

[6] V. Lippiello, J. Cacace, A. Santamaria-Navarro, J. Andrade-Cetto, M. A. Trujillo, Y. R. Esteves, and A. Viguria, 'Hybrid Visual Servoing With Hierarchical Task Composition for Aerial Manipulation', IEEE Robot. Autom. Lett., vol. 1, no. 1, pp. 259-266, Jan. 2016.

[7] T. Adnan, S. Datta, and S. MacLean, 'Efficient and accurate sensor network localization', Pers. Ubiquitous Comput., vol. 18, no. 4, pp. 821-833, Apr. 2014.

[8] Y. Guo and X. Liu, 'A Research on the Localization Technology of Wireless Sensor Networks Employing TI’s CC2530 Instrument’, 2015, pp. 446-449.

[9] G. M. Hoffmann, H. Huang, S. L. Waslander, and C. J. Tomlin, 'Quadrotor helicopter flight dynamics and control: Theory and experiment', in Proc. of the AIAA Guidance, Navigation, and Control Conference, 2007, vol. 2.

[10] R. Mahony, V. Kumar, and P. Corke, 'Multirotor Aerial Vehicles: Modeling, Estimation, and Control of Quadrotor', IEEE Robot. Autom. Mag., vol. 19, no. 3, pp. 20-32, Sep. 2012. 